\title{
Suspected Metallic Embolization Distal to Coiled Intracranial Aneurysms Detectable by Susceptibility-Weighted MR Imaging
}

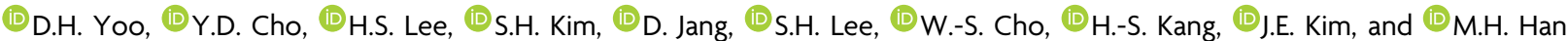

\begin{abstract}
BACKGROUND AND PURPOSE: After endovascular coiling of intracranial aneurysms, round dark parenchymal lesions believed to be particulate metal are sometimes encountered in MR imaging studies of the brain. We used SWI to assess the frequency of such occurrences, in addition to exploring likely causes and clinical implications.
\end{abstract}

MATERIALS AND METHODS: We reviewed 700 MR imaging studies performed between September 2018 and March 2019 at our institution as follow-up monitoring of coiled intracranial aneurysms. Any sizeable $(>5 \mathrm{~mm})$ rounded dark-signal lesions encountered were presumed to be metallic. The magnitudes and locations of such lesions were recorded. In patients with these lesions, pertinent procedural documentation was screened for devices used, including coils, microcatheters, microguidewires, and stents. Medical records were also examined to determine whether any related symptoms ensued.

RESULTS: Twenty patients (2.8\%) exhibited a total of 25 lesions on SWI. Diameters ranged from 5 to $11 \mathrm{~mm}$ (median, $8 \mathrm{~mm}$ ). All except 2 lesions were located in brain regions downstream from aneurysms, but all lesions occupied vascular territories of vessels used to place guiding catheters. Other than the Synchro 14, which was routinely deployed, no device was regularly used in patients with SWI-detectable lesions; and none of the affected patients developed focal neurologic symptoms as a consequence.

CONCLUSIONS: Although the origins remain unclear, distal embolization of particulate metal distal to coiled cerebral aneurysms is occasionally observed on follow-up MR imaging studies. Such lesions, however, seem to have no apparent clinical impact.

ABBREVIATIONS: AcomA = anterior communicating artery; ICA = internal carotid artery; MCA = middle cerebral artery

A lthough endovascular coiling has become an established treatment option for intracranial aneurysms, the relatively high rates of recanalization in initially occluded aneurysms have necessitated periodic follow-up imaging. ${ }^{1,2}$ DSA is still the criterion standard for evaluating saccular occlusions, despite the inherent thromboembolic risk, radiation exposure, and use of contrast. Multiple studies have also found MRA to be a reliable means of monitoring coiled aneurysms. ${ }^{3}$ Furthermore, TOFMRA has the added advantage of no contrast requirement, and axial source images of TOF-MRA may be especially useful in

Received December 10, 2019; accepted after revision February 19, 2020.

From the Departments of Radiology (D.H.Y., Y.D.C., H.S.L., S.H.K., M.H.H.), and Neurosurgery (D.J., S.H.L., W.-S.C., H.-S.K., J.E.K.), Seoul National University Hospital, Seoul National University College of Medicine, Seoul, Korea; and Department of Neurosurgery and Radiology (M.H.H.), Veterans Health Service Medical Center, Seoul, Korea.

Please address correspondence to Young Dae Cho, MD, PhD, Department of Radiology, Seoul National University College of Medicine, 28 Yongon-Dong, Jongno-Gu, Seoul, 110-744, Korea: e-mail: aronnn@naver.com

Indicates article with supplemental on-line table.

http://dx.doi.org/10.3174/ajnr.A6506 screening aneurysms for recurrence. ${ }^{4}$ On inspecting TOF-MRA source images of coiled intracranial aneurysms, we sometimes encounter peculiar, rounded dark-signal lesions (other than the coiled masses themselves) within the brain parenchyma. These lesions are thought to be particulate metal, as previously determined in a series of patients using T1, T2, diffusion, and T2*weighted imaging. ${ }^{5}$

The present study was conducted to evaluate the frequency with which distal embolization of metallic material occurs in conjunction with the coiling of intracranial aneurysms. We also explored potential causes and clinical implications of these lesions. Because the entire brain is not accessible by TOF-MRA, SWI was engaged as well. SWI additionally offers ready visibility of lesions owing to pronounced blooming artifacts, thus heightening the diagnostic sensitivity.

\section{MATERIALS AND METHODS}

This study was approved by our institutional review boards (Seoul National University Hospital). Given the retrospective nature of this study, written informed consent was waived. We 
retrieved and reviewed MR imaging studies performed at our institution (Seoul National University Hospital) between September 2018 and March 2019, each as follow-up in patients with coiled intracranial aneurysms. Our routine institutional follow-up protocol calls for monitoring by TOF-MRA at 6,18 , and 36 months after procedures, with additional interim or subsequent testing as clinically required. Within the study period, SWI was added for improved sensitivity and full coverage of the brain. Multislab TOF-MRA was performed using 3 axial slabs of 40-45 sections per slab at section thickness/overlap of $1.0 \mathrm{~mm} / 50 \%$ or $0.5 \mathrm{~mm} / 0 \%$. Most TOF-MRA images addressed the base of the skull to the mid-Sylvian region, depending on the proximity or remoteness of coiled aneurysms. SWI was acquired at 2.0- to 2.5$\mathrm{mm}$ thickness, extending from the base of the skull to the vertex.

The SWI studies of each patient were first reviewed to check for dark-signal lesions. We presumed that metallic fragments would be rounded and $>5 \mathrm{~mm}$ in diameter. Lesions of irregular shapes or $<4 \mathrm{~mm}$ across were excluded as potential microhemorrhages. If such lesions were detected by SWI, TOF-MRA source images were scrutinized to pinpoint their presence. If available, we also reviewed the following: 1) preprocedural MR imaging studies establishing the procedure-related nature of lesions, 2) other postprocedural MR imaging examinations reflecting temporal changes, and 3) pre- and postprocedural brain CT examinations.

Table 1: Characteristics of treated aneurysms in the study population

\begin{tabular}{lc}
\hline \multicolumn{1}{c}{ Characteristic } & Aneurysm $(\boldsymbol{n}=859)$ \\
\hline Ruptured/unruptured & $78(9.1 \%) / 781(90.9 \%)$ \\
Size of aneurysm & $5.2 \pm 3.1$ \\
Mean (mm) & \\
Distribution (mm) & $222(25.8 \%)$ \\
-3 & $475(55.3 \%)$ \\
$4-6$ & $119(13.9 \%)$ \\
$7-9$ & $43(5.0 \%)$ \\
$10-$ & \\
Location of aneurysm & $363(42.2 \%)$ \\
Internal carotid artery & $193(22.5 \%)$ \\
Anterior cerebral artery & \\
Middle cerebral artery & $207(24.1 \%)$ \\
Vertebrobasilar artery & $96(11.2 \%)$ \\
\hline
\end{tabular}

${ }^{a}$ Including an anterior communicating artery aneurysm.

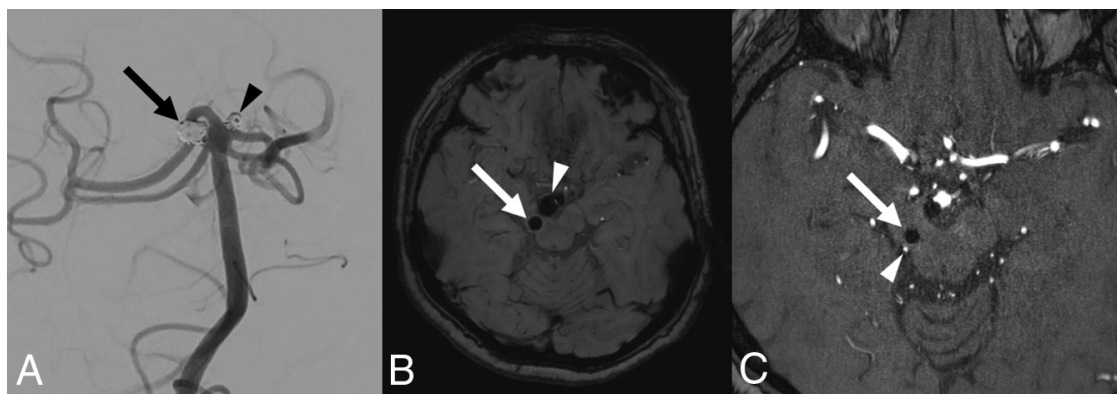

FIG 1. A, Coil embolization of 2 aneurysms involving the right superior cerebellar artery (arrow) and basilar tip (arrowhead). B, SWI 20 months later shows an 8-mm rounded, dark-signal lesion of the right midbrain (arrow) and another dark signal representing a coil mass (arrowhead). C, Source image from TOF-MRA depicts the same lesion, measured at $4 \mathrm{~mm}$ (arrow), with the right superior cerebellar artery (arrowhead) coursing posteriorly.
Most endovascular procedures were conducted with the patient under general anesthesia. Antiplatelet premedication was routinely administered to patients with unruptured aneurysms. A bolus of heparin (3000 IU), administered on placement of the femoral arterial sheath, was thereafter sustained by hourly doses (1000 IU); activated clotting time was monitored each hour. Continuance of dual-antiplatelet therapy was advised for at least 3 months postoperatively, followed by single-agent maintenance for at least 1 year in patients with stents deployed. In the absence of stent placement, antiplatelet therapy was selectively dispensed in instances of prior antiplatelet medication use, coil protrusion, or procedural thromboembolism.

Patient demographics and characteristics of coiled aneurysms, such as size, location, and rupture status, were collected. In patients with dark-signal lesion on SWI, their locations and magnitudes were recorded, with particular attention paid to any restriction within the vascular territories of catheterized arteries. Procedural data were similarly reviewed to identify devices used, including coils, microcatheters, microguidewires, and stents. Finally, medical records were screened for any related patient symptoms.

\section{RESULTS}

MR imaging studies of 700 patients were subject to review. The study population included 189 men (27\%) and 511 women (73\%), with a mean age of 61.4 years (range, $18-86$ years) at the time of MR imaging. In all except 12 patients, a 3T MR imaging unit was used. The interval between the most recent coiling session and current MR imaging ranged from 4 to 177 months (mean, 26.5 months), distributed as follows: $\leq 6$ months, 227 (32.4\%); 7-18 months, 218 (31.2\%); 19-36 months, 133 (19.0\%); and $>36$ months, $122(17.4 \%)$. In this patient population, 859 aneurysms were treated overall, the characteristics of which are detailed in Table 1. Multiple aneurysms were found in 131 patients, and 77 patients were retreated due to recanalization. Twelve patients had additional aneurysms managed by surgical clipping.

A total of 25 dark-signal lesions suspicious for particulate metal were detected by SWI in 20 patients (2.8\%) with 24 treated aneurysms (Figs 1 and 2). Specific features of their treated aneurysms and the dark-signal lesions observed are summarized in Table 2. Although 2 patients (patients 11 and 12) had undergone prior coiling of other aneurysms, no dark-signal lesions had previously resulted. In the 2 instances of retreatment due to recanalization of coiled aneurysms (patients 6 and 16), no dark-signal lesion was detected after initial treatment. The mean procedure-toMR imaging interval was 16 months (range, 4-67 months).

The diameters of dark-signal lesions ranged from 5 to $11 \mathrm{~mm}$ (median, $8 \mathrm{~mm}$ ). Among the 25 lesions identified on SWI, 14 (56\%) were covered in the 


\begin{tabular}{|c|c|c|c|c|c|c|c|}
\hline \multirow[b]{2}{*}{ Case No. } & \multirow[b]{2}{*}{ Age (yr)/Sex } & \multicolumn{2}{|c|}{ Aneurysm } & \multicolumn{3}{|c|}{ Lesion } & \multirow{2}{*}{$\begin{array}{c}\text { Anticoagulant or } \\
\text { Antiplatelet Maintenance }\end{array}$} \\
\hline & & Location & Size (mm) & Location & Size (mm) & TOF-MRA $^{a}$ & \\
\hline 1 & $44 / F$ & Rt ICA & 6 & Rt parietal & 11 & No & Antiplatelet \\
\hline 2 & $57 / \mathrm{M}$ & AcomA & 4 & Lt temporal & 10 & Yes & None \\
\hline \multirow[t]{2}{*}{3} & $72 / \mathrm{M}$ & Lt ICA & 5 & Lt temporal & 10 & Yes & Antiplatelet \\
\hline & & AcomA & 5 & & & & \\
\hline 4 & $72 / \mathrm{M}$ & AcomA & 5 & Rt frontal & 7 & No & Antiplatelet \\
\hline \multirow[t]{2}{*}{5} & $60 / F$ & Rt SCA & 5 & Rt midbrain & 8 & Yes & None \\
\hline & & Lt PCA & 3 & & & & \\
\hline 6 & $68 / F$ & Lt ICA ${ }^{c}$ & 3 & Lt parietal & 11 & No & Antiplatelet \\
\hline 7 & $49 / \mathrm{M}$ & Lt ICA & 4 & Lt temporal & 9 & Yes & Antiplatelet \\
\hline 8 & $63 / F$ & Rt ICA & 4 & Rt temporal & 9 & Yes & None \\
\hline \multirow[t]{2}{*}{9} & $52 / F$ & Rt MCA & 4 & Rt temporal & 10 & Yes & Antiplatelet \\
\hline & & Rt ICA & 4 & Rt CC & 10 & Yes & \\
\hline \multirow[t]{3}{*}{10} & $19 / \mathrm{F}$ & Lt ICA & 15 & Rt frontal & 6 & Yes & Antiplatelet \\
\hline & & & & Lt frontal & 5 & No & \\
\hline & & & & Lt frontal & 5 & No & \\
\hline 11 & $59 / \mathrm{F}$ & Rt ICA & 3 & Lt parietal & 8 & No & None \\
\hline 12 & $66 / F$ & AcomA & 3 & Lt frontal & 10 & No & Antiplatelet \\
\hline 13 & $49 / F$ & Rt ICA & 7 & Rt frontal & 5 & Yes & Antiplatelet \\
\hline \multirow[t]{3}{*}{14} & $39 / F$ & $\mathrm{Lt} I C A^{\mathrm{c}}$ & 4 & Lt BG & 7 & Yes & Antiplatelet \\
\hline & & & & Rt parietal & 7 & Yes & \\
\hline & & & & Rt parietal & 7 & No & \\
\hline 15 & $60 / F$ & Rt MCA & 11 & Rt parietal & 8 & No & None \\
\hline 16 & $62 / F$ & Rt SCA ${ }^{c}$ & 8 & Rt occipital & 10 & Yes & Antiplatelet \\
\hline \multirow[t]{2}{*}{17} & $63 / F$ & Rt ICA & 4 & Rt frontal & 6 & Yes & Antiplatelet \\
\hline & & Lt ICA & 3 & & & & \\
\hline 18 & $71 / \mathrm{M}$ & AcomA & 6 & Rt frontal & 5 & No & Antiplatelet \\
\hline 19 & $54 / F$ & Lt ICA & 4 & Lt CC & 5 & Yes & None \\
\hline 20 & $58 / \mathrm{F}$ & Rt MCA & 5 & Rt frontal & 7 & No & None \\
\hline
\end{tabular}

Note:-Rt indicates right; Lt, left; PCA, posterior cerebral artery; CC, corpus callosum; SCA, superior cerebellar artery; BG, basal ganglia.

${ }^{a}$ Indicates whether the dark-signal lesion on SWI was covered in the source image of TOF-MRA.

${ }^{b}$ Indicates maintenance $>1$ month after coil embolization.

${ }^{\mathrm{c}}$ Denotes ruptured aneurysm.

scan range of TOF-MRA source images (Fig 1). Preprocedural MR imaging studies were available in 11 of 20 patients with dark-signal lesions. No evidence of pre-existing dark-signal lesions was documented in all 11 patients. Postprocedural CT scans of the brain available in 3 patients (patients 9, 14, and 16) were devoid of lesions at corresponding dark-signal locations (not detected on CT). Six patients (patient 5, 8, 15, 17, 18, and 19) underwent earlier postprocedural MR imaging examinations during the follow-up (TOF-MRA in 5 patients, and $\mathrm{T}^{\star}$ weighted imaging in 1 patient) with a mean interval of 21 months (range, 6-49 months). In all 6 patients, there was no change in the lesions at all when compared with the same available sequence of MR images (SWI in case of T2*-weighted imaging).

Except for 2 instances (patients 2 and 15), all lesions occupied regions of the brain downstream from coiled aneurysms. However, all were within vascular territories of vessels used to place guiding catheters. In patient 2, the left ICA served for placement of the guiding catheter during the coiling of an anterior communicating artery (AcomA) aneurysm, leaving a dark-signal lesion in the left MCA territory. In patient 15, the dark-signal lesion appeared within the parasagittal right parietal lobe in the anterior cerebral artery territory after coiling of a right MCA aneurysm. The bilateral anterior cerebral artery territory was solely or predominantly supplied by the right or left ICA in 4 patients (patients 4, 10, 11, and 18), resulting in dark-signal lesions contralateral to coiled aneurysms. In patient 14, the left ICA alone provided bilateral hemispheric perfusion due to chronic right ICA occlusion. Darksignal lesions appeared within the right MCA territory after coiling of a left-sided aneurysm.

No device common to patients with the dark-signal lesions was identified, except for the Synchro 14 (Stryker, Kalamazoo, Michigan), which was routinely deployed in our study population (withheld in 2 patients [0.3\%] only). Devices used for each patient with dark-signal lesions are itemized in the On-line Table. None of the patients with dark-signal lesions developed focal neurologic symptoms attributable to corresponding lesions.

\section{DISCUSSION}

In cerebral angiographic procedures, whether diagnostic or therapeutic, there is a certain risk of cerebral embolism. ${ }^{6}$ Ischemic lesions may be seen by diffusion-weighted imaging in about half of patients after endovascular coiling of intracranial aneurysms, though most are not neurologically evident. ${ }^{7}$ The potential causes of these include air bubbles, thrombi, and disrupted atherosclerotic plaque. A variety of foreign materials have also been implicated. Since the advent of cerebral angiography, reports of embolic cotton or synthetic fibers have surfaced, their origins traced to surgical drapes or gauze. ${ }^{8-10}$ More recently, the hydrophilic polymer coating of catheters and guidewires has become a documented source of downstream emboli following cerebral angiography or interventional procedures. ${ }^{11-14}$ 


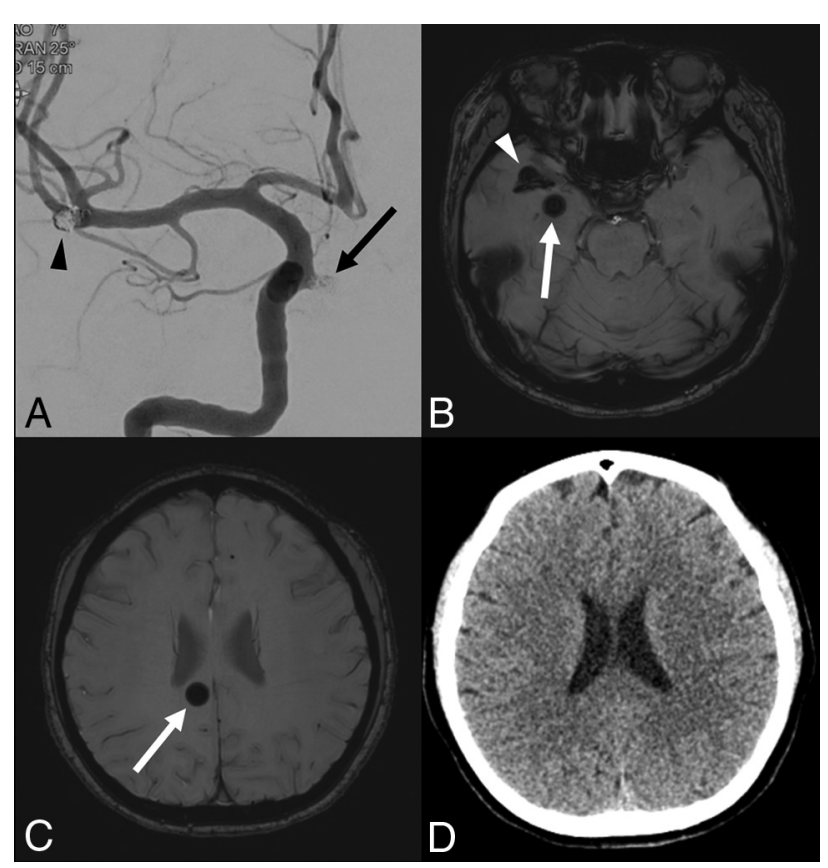

FIG 2. $A$, Coil embolization of 2 aneurysms arising from the right ICA (arrow) and MCA at the bifurcation (arrowhead). B, SWI 6 months later demonstrates a $10-\mathrm{mm}$ rounded dark-signal lesion within right temporal lobe (arrow) and another dark signal representing a coil mass and stent (arrowhead). C, A second rounded dark-signal lesion $(10 \mathrm{~mm})$ is present within the right corpus callosum (arrow). D, The lesion of right corpus callosum is undetectable by postprocedural CT.

Devices intended for interventional procedures contain metallic substances by design to confer fluoroscopic visibility and enhance biostability/durability. Still, reports of suspected particulate metal emboli after endovascular treatment of intracranial aneurysms or cardiac catheterization have been surprisingly few. ${ }^{5}$ In the present study, suspicious metallic emboli were identified by MR imaging in $2.8 \%$ of a sizeable patient population $(n=700)$ with coiled intracranial aneurysms. This figure is somewhat lower than that cited in a previous study, in which $5.5 \%$ of patients $(6 / 110)$ displayed metallic embolic phenomena in postprocedural MR imaging studies under similar circumstances. ${ }^{5}$

Most dark-signal lesions encountered herein fell within vascular territories of vessels used to place guiding catheters. The exceptions in 2 instances suggest that such embolization is not always linked to coil deployment/detachment or positioning of stents during endovascular procedures. Instead, it may occur while navigating to or from aneurysms, gaining access or retrieving devices. The only device shared by patients with dark-signal lesions was the Synchro 14 microwire (Stryker). We have often found that microguidewire coatings may be peeled off as torque is applied, serving as a potential cause of dark-signal lesions (microfragments of the coating). However, the Synchro 14 was used in essentially all of our procedures, so it can hardly be considered the culprit device.

Coil detachment may be achieved in a variety of ways, including mechanical, electrolytic, electrothermal, and hydraulic methods. When the present study was conceived, we wondered whether some coil-detachment strategies might precipitate micrometallic fragmentation. However, our data have dispelled any relation between dark-signal lesions and a single detachment system, and no other devices (ie, guiding catheters, microcatheters, or stents) were consistently used in the presence of dark-signal lesions.

Previous reports on inadvertent distal embolization of foreign matter have confirmed subsequent neurologic symptoms due to hemorrhage or focal edema, ${ }^{11,13,14}$ whereas we encountered no clinical manifestations owing to dark-signal lesions. In addition, FLAIR images available in 3 patients with dark-signal lesions revealed no abnormal signal around the lesion. It is feasible that, despite their various sizeable appearance on SWI, such minute fragments of metal, small enough to escape detection by CT, are too small to induce embolic infarction. In addition, these lesions were unchanged with time in 6 patients, underscoring the relative biostability of such emboli and the likely absence of foreign body reaction.

Our study has several acknowledged limitations. First, the dark-signal lesions present on SWI were never pathologically confirmed as metallic in nature because none of the patients had clinical conditions calling for biopsy. Furthermore, preprocedural MR imaging and immediate postprocedural MR imaging were not available in all patients, so we could not conclusively ascertain whether all dark-signal lesions were procedure-related. The possibility of pre-existing hemorrhage or calcification still remained. In addition, patients who underwent stent-assisted coiling routinely received antiplatelet medication for at least a year, which might increase incidence of microhemorrhage. ${ }^{15}$ Another pitfall was the potential to underestimate the incidence of metallic emboli by excluding lesions of $<4 \mathrm{~mm}$ or by failing to detect lesions near the base of the skull, the latter explained by susceptibility artifacts from bone and air. Finally, our study was retrospective by design, incorporating $\mathrm{MR}$ imaging protocols and follow-up intervals that were not consistent within the study population. A prospective investigation involving controlled perioperative MR imaging studies would perhaps yield more reliable results.

\section{CONCLUSIONS}

Although a causative device or mechanism has yet to be implicated, embolization of particulate metal distal to coiled cerebral aneurysms is not an infrequent finding in follow-up MR imaging studies. In this setting, however, the patients seem unaffected clinically.

Disclosures: Moon Hee Han—UNRELATED: Consultancy: MicroVention. * ${ }^{*}$ Money paid to the institution.

\section{REFERENCES}

1. Molyneux A, Kerr R, Stratton I, et al. International Subarachnoid Aneurysm Trial (ISAT) of neurosurgical clipping versus endovascular coiling in 2143 patients with ruptured intracranial aneurysms: a randomised trial. Lancet 2002;360:1267-74 CrossRef CrossRef Medline

2. Lecler A, Raymond J, Rodriguez-Regent C, et al. Intracranial aneurysms: recurrences more than 10 years after endovascular treatment-a prospective cohort study, systematic review, and metaanalysis. Radiology 2015;277:173-80 CrossRef Medline

3. Ahmed SU, Mocco J, Zhang X, et al. MRA versus DSA for the followup imaging of intracranial aneurysms treated using endovascular 
techniques: a meta-analysis. J Neurointerv Surg 2019;11:1009-14 CrossRef Medline

4. Cho YD, Kim KM, Lee WJ, et al. Time-of-flight magnetic resonance angiography for follow-up of coil embolization with Enterprise stent for intracranial aneurysm: usefulness of source images. Korean $J$ Radiol 2014;15:161-68 CrossRef Medline

5. Yasuda R, Maeda M, Umino M, et al. Suspected metallic embolism following endovascular treatment of intracranial aneurysms. AJNR Am J Neuroradiol 2016;37:1696-99 CrossRef Medline

6. Bendszus M, Koltzenburg M, Burger R, et al. Silent embolism in diagnostic cerebral angiography and neurointerventional procedures: a prospective study. Lancet 1999;354:1594-97 CrossRef Medline

7. Bond KM, Brinjikji W, Murad MH, et al. Diffusion-weighted imaging-detected ischemic lesions following endovascular treatment of cerebral aneurysms: a systematic review and meta-analysis. AJNR Am J Neuroradiol 2017;38:304-09 CrossRef Medline

8. Silberman J, Cravioto H, Feigin I. Foreign body emboli following carotid angiography. Arch Neurol 1960;3:711-17 CrossRef

9. Chason JL, Landers JW, Swanson RE. Cotton fiber embolism: a frequent complication of cerebral angiography. Neurology 1963;13:55860 CrossRef CrossRef Medline
10. Shannon P, Billbao JM, Marotta T, et al. Inadvertent foreign body embolization in diagnostic and therapeutic cerebral angiography. AJNR Am J Neuroradiol 2006;27:278-82 Medline

11. Shapiro M, Ollenschleger MD, Baccin C, et al. Foreign body emboli following cerebrovascular interventions: clinical, radiographic, and histopathologic features. AJNR Am J Neuroradiol 2015;36:2121-26 CrossRef Medline

12. Oh SW, Shin NY, Lee HJ, et al. Delayed enhancing lesions after coil embolization of aneurysms: clinical experience and benchtop analyses. J Neurointerv Surg 2017;9:1243-47 CrossRef Medline

13. Ansari SA, Anderson RR, Caron MJ, et al. Hydrophilic polymer embolic complication during diagnostic cerebral angiography presenting with delayed intracranial hemorrhage: case report and literature review. J Neurointerv Surg 2019;11:80-83 CrossRef Medline

14. Geisbush TR, Marks MP, Heit JJ. Cerebral foreign body reaction due to hydrophilic polymer embolization following aneurysm treatment by Pipeline flow diversion device. Interv Neuroradiol 2019;25:447-53 CrossRef Medline

15. Qiu J, Ye H, Wang J, et al. Antiplatelet therapy, cerebral microbleeds, and intracerebral hemorrhage: a meta-analysis. Stroke 2018;49:1751-54 CrossRef Medline 